\title{
A Novel Precursor Ion Discovery Method on a Hybrid Quadrupole Orthogonal Acceleration Time-of-Flight (Q-TOF) Mass Spectrometer for Studying Protein Phosphorylation
}

\author{
R. H. Bateman, R. Carruthers, J. B. Hoyes, C. Jones, J. I. Langridge, \\ A. Millar, and J. P. C. Vissers \\ Micromass Ltd., Floats Road, Manchester, M23 9LZ, UK
}

\begin{abstract}
A tandem quadrupole time-of-flight (Q-TOF) mass spectrometer has been programmed such that phosphorylated peptides can automatically be discovered and identified in a way similar to that of the use of precursor ion or neutral loss scanning, but without the need to scan the quadrupole mass filter. Instead, the method capitalizes on the innate capability of the Q-TOF to record mass spectra and product ion spectra quickly, with good sensitivity and with good mass accuracy. Alternate mass spectra, with and without fragmentation, are recorded at high and low collision energy with the quadrupole operating in wideband mode. The method of analysis is both compatible with and dependant on liquid chromatography for separation of complex mixtures. The method has been demonstrated by searching for the neutral loss of 98 $\mathrm{Da}\left(\mathrm{H}_{3} \mathrm{PO}_{4}\right)$ from phosphoserine and phosphothreonine residues, or for the phosphorylated immonium ion at $\mathrm{m} / \mathrm{z} 216$ from phosphotyrosine. The method also incorporates acquisition of the product ion spectrum from any candidate precursor ions, thereby allowing confirmation of the neutral loss or product ion and providing additional sequence information to assist identification of the protein and assign the site of phosphorylation. (J Am Soc Mass Spectrom 2002, 13, 792-803) @ 2002 American Society for Mass Spectrometry
\end{abstract}

$\mathrm{T}$ The decoding of the 30-40 thousand genes that make up the human proteome has made the large-scale identification of biologically relevant human proteins by a combination of separation technologies and mass spectrometry a reality. Isolated or partially isolated proteins are enzymatically digested and the peptide products are analyzed by mass spectrometry. Proteins may be identified directly from their peptide fingerprint by comparing the peptide masses to those expected from a library of known proteins [1]. The more accurate the mass assignments are the more specific the protein identification [2]. The peptide masses may be determined directly from the mass analysis of the mixture using matrix assisted laser desorption ionization (MALDI). Alternatively the components of the mixture may be first separated or partially separated by liquid chromatography before mass analysis using electrospray ionization (ESI). This latter approach allows analysis and identification over a wider dynamic range. When the results of this compar-

Published online June 11, 2002

Address reprint requests to Robert $\mathrm{H}$. Bateman, Micromass Ltd., Floats Road, Wythenshawe, Manchester, M23 9LZ, United Kingdom. E-mail: bob.bateman@micromass.co.uk ison suggest more than one possible protein then the ambiguity can be resolved by analysis of the fragments of one or more of the peptides. Not only does analysis of fragment ion masses from individual peptides confirm the protein identification it also allows identification of proteins from genomic databases.

It is thought that the majority of the proteins present in the human proteome have the potential to be posttranslationally modified, and yet the biological relevance of those modifications is still not well understood. Characterizing and understanding the role of modifications in biological processes and during the onset and progression of disease is of growing interest. Mass analysis of fragment ions from individual peptides by MS/MS allows analysis of the post-translational modifications present on identified proteins. However, the initial discovery of the presence of posttranslational modifications on unidentified proteins, and their subsequent analysis, is a more challenging problem.

Protein phosphorylation is one of the most common post-translational modifications to occur in mammalian systems [3]. It is important in a number of key biological processes, including intracellular signalling and facilitating the action of several key hormones [4,5]. The 
regulation of enzyme activity is often modulated either through the phosphorylation or dephosphorylation of specific protein kinases. This is regulated by specific protein kinases and phosphatases that have differing specificity dependent upon the amino acid residue being modified. Three amino acids, serine, threonine and tyrosine, are commonly modified by the addition of $\mathrm{HPO}_{3}$ to their side chain. This gives rise to an increase in the molecular mass of the peptide by $80 \mathrm{Da}$ for each modified residue.

Phosphorylation of specific protein kinases in cell signaling provides a mechanism for intracellular signal amplification, where each subsequent kinase phosphorylated can in turn target a larger number of protein substrates. Due to the transient nature of cellular signaling events, the lifetime of specific phosphorylation events can be short, thereby adding to the analytical difficulties already faced. A further problem is encountered due to the endogenous levels of protein that are phosphorylated in many phosphorylation mediated mechanisms. As little as $10 \%$ of the available protein may be phosphorylated and yet can still have the relevant biological effect, whilst the presence of large amounts of the non-phosphorylated species provides an obvious dynamic range problem for the analytical approach used to study these events.

Mass spectrometry has started to play a larger role in the study of protein phosphorylation. The MALDI mass spectra of phosphorylated peptides can exhibit fragment ions 80 daltons less than that of the $(\mathrm{M}+\mathrm{H})^{+}$ion resulting from the neutral loss of phosphoric acid from a phosphoserine or phosphothreonine residue [6]. Phosphorylated peptides have also been recognized from their shift in mass following removal of the phosphoric acid group by treatment of the peptide mixture with alkaline phosphatase [7]. In either case phosphorylation of the peptide has been confirmed and the peptide identified by recording its product ion spectrum, for example by post source decay (PSD) analysis on a MALDI-TOF mass spectrometer.

An alternative strategy that has proven highly successful utilizes electrospray ionization in combination with a tandem (MS/MS) mass spectrometer, and makes use of the 'precursor ion' and 'neutral loss' scanning techniques. Molecules that belong to a particular class can sometimes be recognized from a common diagnostic fragment ion or neutral loss and may be discovered by the methods of 'precursor ion' scanning or 'neutral loss' scanning $[8,9,10,11]$. When performing a precursor ion scanning experiment the mass spectrometer searches for all ions that fragment to produce a common, diagnostic product ion. Similarly, when performing a 'neutral loss' scanning experiment the spectrometer searches for all ions that fragment to lose a common, diagnostic neutral.

The 'precursor ion' scan on a triple quadrupole has been used for the discovery of phoshorylated peptides. The negative fragment ion $\mathrm{PO}_{3}^{-}$at $\mathrm{m} / \mathrm{z} 79$ is characteristic of phoshorylated peptides, and the precursor ion scan of this negative fragment ion is now an established method of discovering such peptides [8].

The most common methods for recording precursor ion scans or 'neutral loss' scans involve scanning the first or both quadrupole mass filters in a triple quadrupole mass spectrometer. In the precursor ion scan the first quadrupole mass filter is scanned, while the second quadrupole mass filter is set to transmit just the required product ion. Thus, although the duty cycle (duty cycle can be defined as the percentage of time a mass analyzer spends transmitting those ions of interest during a single experimental cycle.) for the second quadrupole mass filter is $100 \%$, the duty cycle for the first quadrupole mass filter is low, typically of the order of $0.1 \%$. This is similar for the 'neutral loss' experiment, even though in this experiment both quadrupole mass filters are scanned. This is because the second quadrupole mass filter scans in synchronism with the first quadrupole such as to transmit just ions with the required mass difference from that transmitted by the first mass filter. So again the duty cycle for each of the quadrupole mass filters is typically of the order of $0.1 \%$ and $100 \%$ respectively. As a consequence of this poor duty cycle the sensitivity and acquisition rate is such that these methods are not routinely used for the analysis of sub-picomole quantities of peptides by liquid chromatography directly coupled to mass spectrometry.

If it is required to identify the peptide and ascertain the site of phosphorylation it is then necessary to record the product ion spectrum of the candidate precursor ion. This also requires that the second quadrupole mass filter be scanned, again with a low duty cycle of the order of $0.1 \%$. Consequently there is little or no opportunity to confirm and identify a precursor ion by subsequent acquisition of its product ion spectrum within the same LC run. Furthermore other valuable information may be lost whilst the mass spectrometer is occupied recording the precursor ion or 'neutral loss' spectrum.

The orthogonal acceleration time-of-flight hybrid mass spectrometer (oa-TOF) simultaneously samples all masses, and its sensitivity is higher than that of a quadrupole when a full scan is recorded [12]. This is because the oa-TOF has a relatively high duty cycle, typically 5 to $25 \%$, compared to that of a scanning quadrupole mass spectrometer. The tandem quadrupole orthogonal acceleration time-of-flight mass spectrometer (Q-TOF) similarly has a relatively high duty cycle [13], irrespective of whether the spectrum to be recorded is that of precursor ions from the source (MS), or fragment ions from a precursor ion selected by the quadrupole mass filter and fragmented in the collision cell (MS/MS). However, when only a single specified product ion is to be monitored, as in precursor ion scanning or in the multiple reaction monitoring (MRM) experiments, the Q-TOF type instrument is less sensitive than the equivalent triple quadrupole instrument [14]. This is mainly because the duty cycle of the oa-TOF 
remains at 5 to $25 \%$, whereas that of a static quadrupole mass filter increases to $100 \%$.

In comparison to a triple quadrupole mass spectrometer, the Q-TOF type instrument generally has significantly better sensitivity and speed of acquisition when recording mass spectra and product ion spectra, but significantly poorer sensitivity and speed of acquisition when used to record precursor ion and 'neutral loss' spectra.

In conventional oa-TOF operation ions continually stream into the TOF accelerator and are pulsed into the TOF and analyzed. To avoid artefacts in the spectrum the ions must be allowed to travel through to the detector before the accelerator can pulse again and admit the next packet of ions into the analyzer. Ions are lost during this waiting time leading to the 5 to $25 \%$ duty cycle quoted above. A method of trapping ions in an ion guide and releasing them in pulses has been proposed to improve the duty cycle of an oa-TOF [15]. A hybrid quadrupole TOF instrument has been adapted to use this method to enhance the duty cycle for precursor ion scanning experiments [16, 17]. While ions are flying through the TOF to the detector the ions that would have been lost are accumulated in the collision cell. A packet of the accumulated ions is then released for a short period of time (say a few microseconds) by pulsing the exit electrode of the collision cell and after a delay equal to the ion flight time from the gas cell to the TOF accelerator, ions are pushed into the TOF and analyzed. By appropriate setting of the delay of TOF injection pulses with respect to the gating pulse, a $100 \%$ duty cycle can be achieved over a narrow mass range. This is comparable with that of the second quadrupole mass filter in a triple quadrupole instrument. During a precursor ion scan, as the quadrupole mass filter is scanned the transmitted ions are fragmented and trapped in the gas cell. Following release of these ions the TOF is pulsed after a delay optimized for best transmission of the characteristic fragment ion. Nevertheless, it is still necessary to scan the quadrupole mass filter (MS1), and so the duty cycle for this arrangement remains at values of the order of $0.1 \%$.

\section{Theory}

Even though in conventional operation the duty cycle for an orthogonal TOF is less than that for a quadrupole mass filter when monitoring a single mass, it nevertheless records full product ion spectra. When used to emulate precursor ion and 'neutral loss' scanning experiments only the data relating to the characteristic product ion or neutral loss ion is of interest; the rest of the data is discarded. However, a Q-TOF type instrument can quickly acquire the conventional mass spectrum, and this provides a record of all the precursor ions present at that time. This information alone can provide a means of significantly improving the duty cycle of the first quadrupole mass filter. In the subsequent analysis, knowledge of all the precursor ions present allows the Q-TOF instrument to be programmed to record just the product ion spectra of those precursor ions and not that of every possible precursor ion over an arbitrarily pre-selected mass range. If the sample mixture is initially separated by liquid chromatography the number of precursor ions present at any one time may be relatively few. The fewer co-eluting components there are the greater the reduction in the number of product ion spectra to be recorded. For example, if a precursor ion scan were required to discover possible precursor ions over a mass range from $\mathrm{m} / \mathrm{z} 200$ to $\mathrm{m} / \mathrm{z} 1000$, this could be accomplished on a Q-TOF type instrument by programming it to record 800 product ion spectra from 800 different precursor ions each spanning one mass number. Alternatively, the Q-TOF could first be programmed to record the mass spectrum to reveal all the possible precursor ions, and then record the product ion spectrum for each of these possible precursor ions. If the mass spectrum revealed a total of 10 possible precursor ions, then only 10 product ion spectra need be recorded. This is a total of only 11 spectra compared to 800 spectra, i.e., only $1.4 \%$ of the time required. Clearly the advantage to be gained from this approach depends on the complexity of the mixture eluting from the liquid chromatogram at any instant in time.

The number of product ion spectra to be recorded may be further reduced by first testing to determine whether or not the characteristic product ion or neutral loss is present, regardless of the identity of the precursor ion. This may be tested by acquisition of the composite product ion spectrum from all precursor ions from the whole mass spectrum. This can be easily accomplished on a Q-TOF type instrument by operating the quadrupole mass filter in the RF-only mode such as to simultaneously transmit approximately a decade in mass into the gas collision cell with higher collision energy, sufficient to induce fragmentation. All ions from the source with $\mathrm{m} / \mathrm{z}$ values above the low mass cut-off value, 0.78 times the set $\mathrm{m} / \mathrm{z}$ value, of the RF-only quadrupole are transported through the quadrupole and fragmented in the gas collision cell. The orthogonal TOF mass spectrometer records the mass spectrum of the resulting mixture of precursor and fragment ions. It is only necessary to switch to low collision energy to prevent fragmentation and hence to record the mass spectrum of precursor ions only; it is not necessary to remove the gas from the collision cell. Hence, by alternating the collision energy, it is possible to alternate between recording the spectrum exhibiting mainly precursor ions and the spectrum exhibiting the mixture of precursor ions and their fragment ions.

The operating conditions are set such that alternate spectra are recorded under at least two distinct operating modes. In the first mode where the collision energy is low, typically less than 10 volts, none or very few of the precursor ions are fragmented. In the second mode where the collision energy is higher, typically 30 to 70 volts, many or most of the precursor ions are frag- 
mented to produce product ions. If the two operating modes are suitably set, precursor and product ions may be readily distinguished since precursor ions will be relatively more intense in the mass spectrum without fragmentation (first mode) and product ions will be relatively more intense in the mass spectrum with fragmentation (second mode).

Recognition of characteristic product ions can be further aided by setting the low mass cut-off value of the RF-only quadrupole to an $m / z$ value a little higher than that of the characteristic product ion. The low mass cut-off value may be set by appropriate selection of the amplitude of RF voltage applied to the quadrupole. If this is set just above the $m / z$ value of the characteristic product ion this will ensure any background ions from the source at that $m / z$ value will not be transmitted to the collision cell. Hence, any ions observed at this $\mathrm{m} / \mathrm{z}$ value can only be formed in the collision cell. In other words, they can only be product ions.

Precursor ion discovery based on the presence of a characteristic product ion at a specific $m / z$ value requires initial interrogation of only the mass spectrum with fragmentation (second mode). When a product ion of interest is observed the neighboring mass spectra without fragmentation (first mode) now yield the short list of candidate precursor ions. This may be further filtered by the application of additional criteria, such as preferred charge state and excluded $m / z$ values. Confirmation and identification of the targeted precursor ion now only requires acquisition of product ion spectra for the short list of candidates. This achieves the same goal as traditional 'precursor ion scanning' without the need to scan MS1, and with the added bonus of having acquired the full product ion spectrum of the targeted precursor ion.

Precursor ion discovery based on the presence of a characteristic neutral loss requires interrogation of both sets of mass spectra, with and without fragmentation. If the spectra exhibit precursor and product ions with a mass difference corresponding to that of the neutral loss then the precursor ion can be entered into a short list of candidate precursor ions. Again this short list may be further filtered by additional criteria, such as preferred charge state and excluded $\mathrm{m} / \mathrm{z}$ values. Any candidate precursor ion may be confirmed and identified by acquisition of its product ion spectrum. This achieves the same goal as traditional 'neutral loss scanning' without the need to scan MS1, and again with the added bonus of having acquired the full product ion spectrum of the targeted precursor ion.

The number of candidate precursor ions is further reduced if exact $m / z$ values, rather than nominal values, can be specified for characteristic product ions and characteristic neutral loss mass differences. This increase in specificity can be particularly beneficial to precursor ion discovery based on the presence of a characteristic neutral loss. For example, in the case of phosphorylated peptides both phosphoserine and phosphothreonine containing precursor ions may be identified by the neutral loss of $\mathrm{H}_{3} \mathrm{PO}_{4}$, i.e., by a difference in $\mathrm{m} / \mathrm{z}$ value between precursor and product ions of 97.977 for singly charged ions, or 48.989 for doubly charged ions. This mass difference is markedly mass deficient compared to other likely neutral losses with a nominal mass difference of 98 daltons. Phosphotyrosine containing peptides may be identified by the detection of the characteristic low-mass immonium ion at $m / z$ 216.043. Clearly $m / z$ values should be specified with tolerances compatible with the mass measurement accuracy to be expected from the mass spectrometer.

Here the application is to analyze a protein digest mixture searching only for those peptides that are potentially phosphorylated. We describe an experimental methodology that allows specific post-translationally modified peptides to be automatically discovered and identified during the course of a single HPLC experiment on a Q-TOF type mass spectrometer.

\section{Experimental}

\section{Chemicals}

All chemicals unless otherwise stated were purchased from Sigma Chemical Co. (Poole, Dorset, UK). Acetonitrile was purchased from Riedel-de-Haen $\mathrm{GmbH}$ whilst HPLC grade water was obtained from Fisher Scientific (Loughborough, UK). PepMap HPLC columns were obtained from LC-Packings (Amsterdam, The Netherlands) while Symmetry C18 columns were obtained from Waters (Milford, MA, USA). Casein protein samples were obtained from Sigma (Poole, Dorset, UK). The phosphopeptide samples were diluted to the required concentrations in $0.1 \%$ formic acid aqueous solution and 1 to $5 \mu \mathrm{L}$ of the resulting solution were used for analysis by LC-MS/MS.

\section{Sample Preparation-Tryptic Digestion}

Intact protein samples $(250 \mu \mathrm{g})$ were solubilized in 100 $\mathrm{mM}$ ammonium bicarbonate and tryptic digests performed by the addition of $12.5 \mu \mathrm{g}$ of TPCK treated trypsin (Sigma, Poole, Dorset) in $100 \mathrm{mM}$ ammonium bicarbonate. The reaction was allowed to proceed at a temperature of $37^{\circ} \mathrm{C}$ for three hours with the enzymatic reaction halted by freezing aliquots at $-20^{\circ} \mathrm{C}$. The tryptic peptides were then diluted to the stated concentration prior to analysis, in $0.1 \%$ aqueous formic acid.

\section{LC-MS/MS}

The samples were injected into a Micromass CapLC liquid chromatography system (Micromass, Manchester, UK), at a flow rate of $30 \mu \mathrm{L} / \mathrm{min}$, and pre-concentrated on a $320 \mu \mathrm{m} \times 5 \mathrm{~mm}$ PepMap C18 precolumn. The flow from pump $C$ was used to load the sample and wash the sample for 3 minutes with a $0.1 \%$ formic acid aqueous solution. The peptides were then eluted onto an analytical C18 column (150 mm $\times 75 \mu \mathrm{m})$. The 


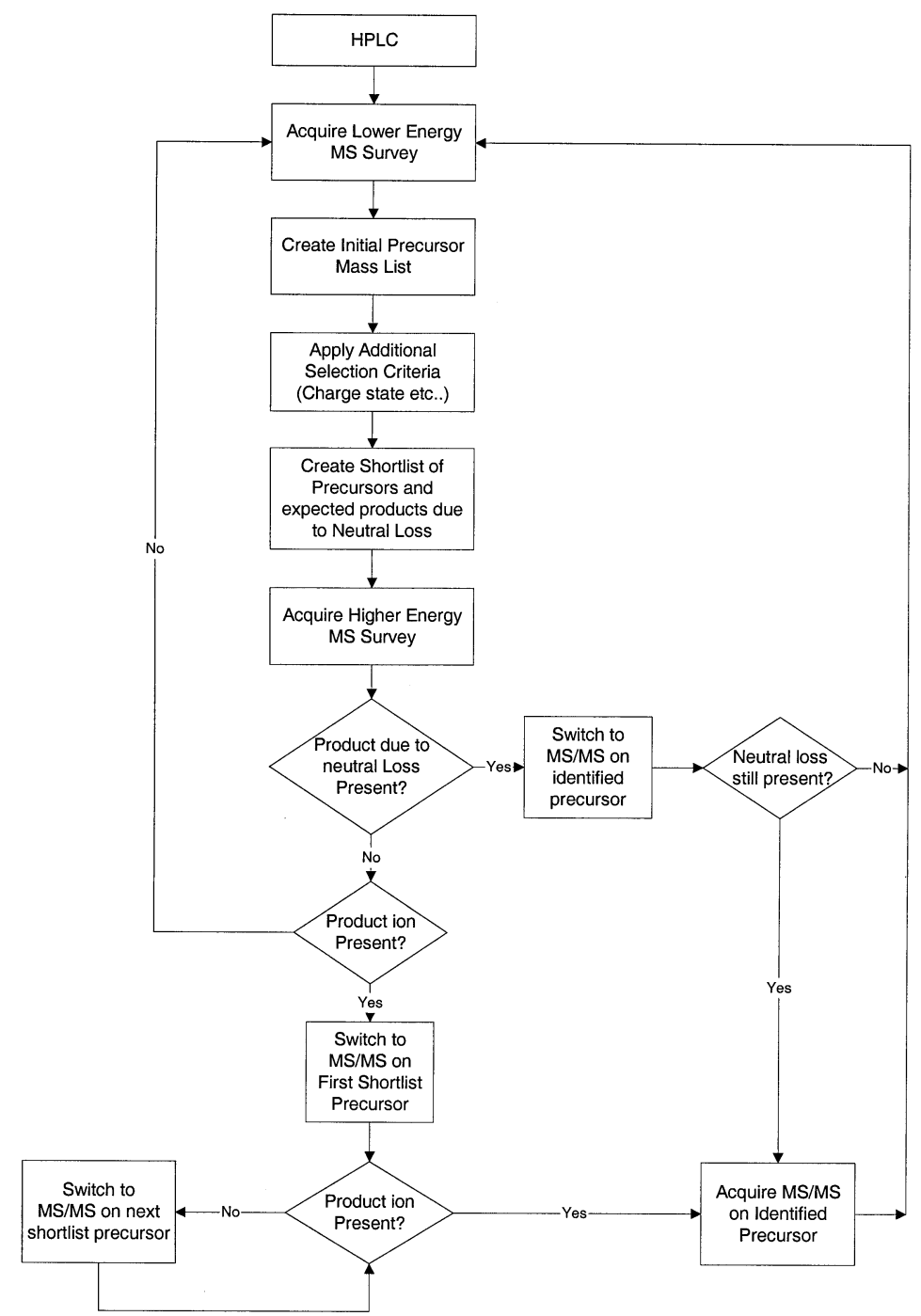

Figure 1. Simplified flow diagram showing the sequence of events leading to the discovery of a precursor ion from a pre-defined neutral loss or product ion.

chromatography separation used a gradient from Solution A, (5\% acetonitrile: $95 \%$ water) to Solution B ( $40 \%$ acetonitrile: $60 \%$ water), both containing $0.1 \%$ formic acid, over 40 minutes at a flow rate of $200 \mathrm{~nL} / \mathrm{min}$. The flow from pumps A and B was $2 \mu \mathrm{L} / \mathrm{min}$, and this was split to reduce the flow rate through the column to approximately $200 \mathrm{~nL} / \mathrm{min}$.

The LC eluent from this column was directed to the nanoflow source on either a Q-TOF 2 or Q-TOF Ultima API mass spectrometer (Micromass, Manchester, UK). The mass spectrometer was operated in positive ion mode with a source temperature of $80^{\circ} \mathrm{C}$ and a cone gas flow of $50 \mathrm{~L} / \mathrm{hr}$. A voltage of $3.0-3.5 \mathrm{kV}$ was applied to the nanoflow probe tip. Mass spectra were acquired with the oa-TOF analyser in the V-mode of operation [18], and spectra were integrated over one second intervals. The quadrupole RF was set to give a low cut-off, which also gave efficient transmission of ions upto the maximum mass of acquisition. MS data were acquired in centroid mode and MS/MS data acquired in continuum mode, and all data were processed using MassLynx version 3.5 software. The instrument was calibrated with a multi-point calibration using selected fragment ions that resulted from the collision-induced decomposition (CID) of Glu-fibrinopeptide B.

\section{Method}

During the HPLC gradient the mass spectrometer continuously switched back and forth between the two survey modes, each with argon in the gas cell, with the pressure approximately set at $1 \times 10^{-3}$ mbar, but with two different collision energies, until certain criteria were met indicating the presence of a phospylated peptide. In both survey modes the quadrupole mass filter (MS1) was operated in the RF only mode allowing a wide mass range to be transmitted to the TOF. The quadrupole RF voltage was set such that it did not transmit ions with $\mathrm{m} / \mathrm{z}$ values below 400 . Spectra from the first survey mode were acquired at low collision 

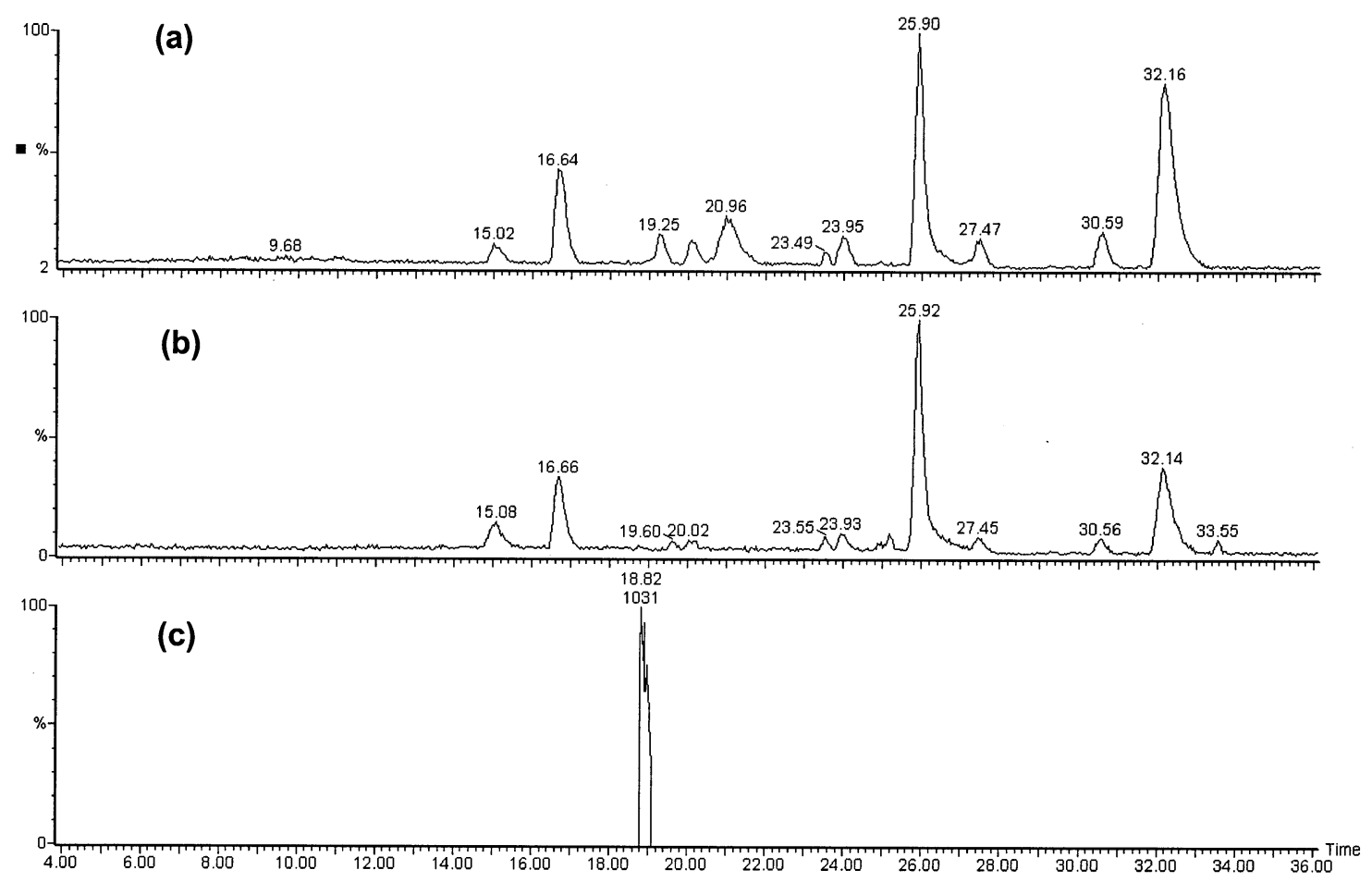

Figure 2. The base peak intensity (BPI) chromatograms obtained from the LC-MS precursor ion discovery analysis of $100 \mathrm{fmol}$ of a beta casein tryptic digest. The chromatograms show the lower (a) and higher (b) collision energy data sets, while trace (c) represents the MS/MS BPI chromatogram. In this example only one peak $\mathrm{m} / z 1031.4(2+)$ exhibited a neutral loss of $\mathrm{H}_{3} \mathrm{PO}_{4}(48.9879 \mathrm{Da})$ within the specified mass tolerance of $\pm 0.020 \mathrm{Da}$ and was therefore the only ion selected for fragmentation in the product ion mode.

energy, typically with not more than 10 volts applied to the collision cell, and showed only pseudo molecular precursor ions. The collision energy was increased for the second survey mode so that fragment ions were generated from the precursor ions present in the first survey mode spectrum. Fragment ions with $m / z$ values less than 400 are free of any interference from source and LC chemical background that would otherwise be present. A list of candidate precursors was generated from the first, low energy survey mode spectra. The second, higher energy survey mode spectra were interrogated for the presence of the predefined neutral loss and/or product ions. When detected, the instrument automatically switched to record the product ion spectrum of the candidate precursor ion or ions to confirm or otherwise that the chosen precursor gave rise to the observed neutral loss or product. If confirmed, the instrument may remain in the MS/MS mode to acquire more sequence data before reverting to acquisition of alternate spectra in the two survey modes. It should be noted that this method relies on the ability of the LC system to concentrate and separate analytes of interest so that they stand out as distinct peaks above the background.

The two cases of neutral loss and product ion generation are quite distinct. In the case of a neutral loss there is only one suspected precursor, that which gave rise to the calculated neutral loss, and so switching to
MS/MS will immediately reveal a successful hit or a false positive. On the other hand the product ion is not tagged with any particular precursor mass and so discovery takes place by process of elimination as the instruments switches sequentially on the precursor shortlist. Figure 1 is a simplified flow diagram illustrating the sequence of events leading to a discovery of precursor ions from pre-defined neutral losses and product ions. It should be noted that the experiment may be configured to look for neutral losses only, product ions only, or both species simultaneously.

Even with chromatographic separation the low energy MS survey spectra can be very complex and certain other predetermined criteria were required to be met before the $m / z$ value was entered into the short list of candidate precursors. Here candidate precursor ion selection was further limited to those that exhibited doubly charged ions.

In the case of phosphopeptides, both phosphoserine and phosphothreonine containing precursors may be identified as they display a neutral loss of $98 \mathrm{Da}$ $\left(\mathrm{H}_{3} \mathrm{PO}_{4}\right)$ under the higher collision energy conditions. Correspondingly, the software made a list of neutral losses from the precursors identified in the lower collision energy spectrum. This involved measuring the masses of the precursor ions, determining their charge states and subtracting the neutral loss i.e., 97.977 for singly charged ions and 48.989 for doubly charged ions. 

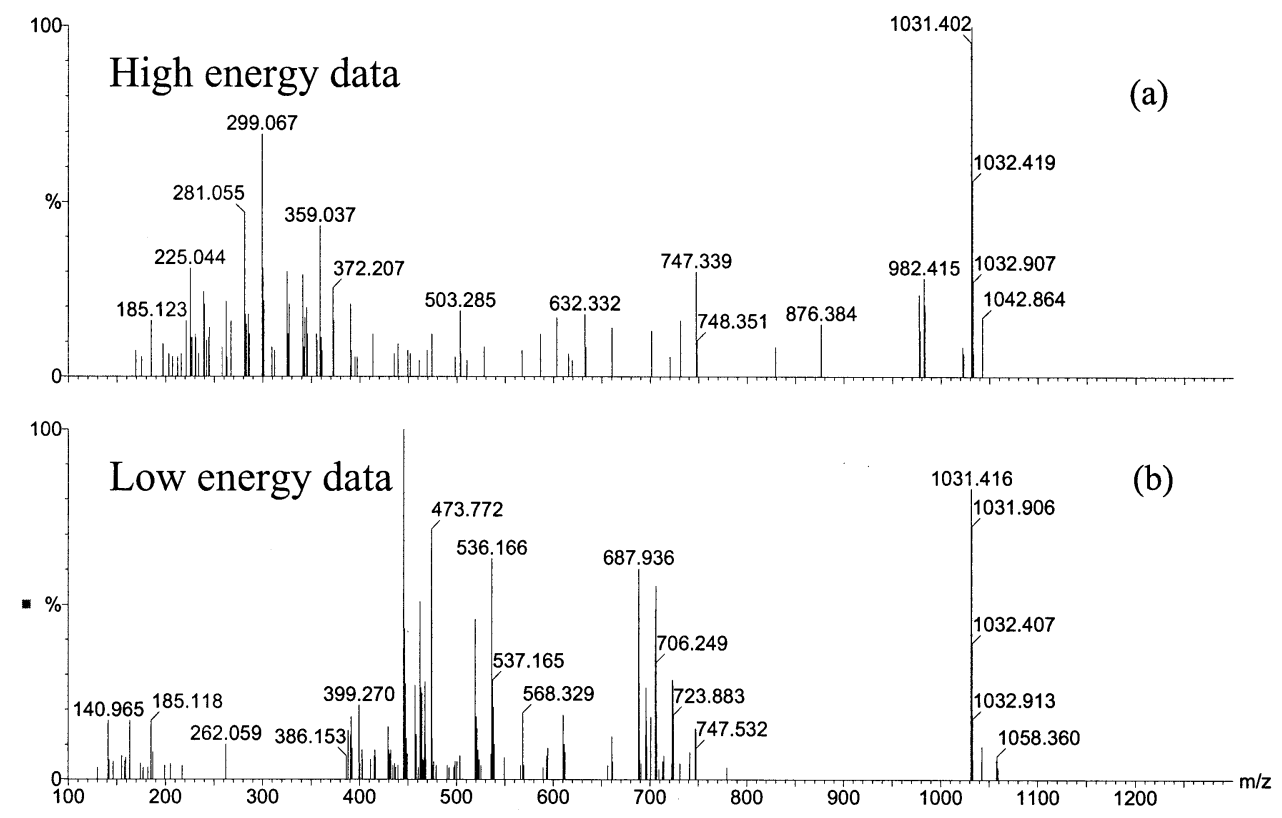

Figure 3. The higher $(32 \mathrm{~V})$ and lower $(10 \mathrm{~V})$ collision energy mass spectra at the time of elution of the doubly charged ion at $m / z$ 1031.4. The co-eluting ions present in the low energy mass spectrum (b), gave rise to the associated set of fragments present in the high energy spectrum (a).

As shown in the flowchart the appearance of the neutral loss in the higher collision energy spectrum caused the instrument to switch into the product ion mode to confirm the neutral loss and to acquire additional sequence information. The exact mass capability of the Q-TOF mass spectrometer allowed the neutral loss to be specified to within $\pm 20 \mathrm{mDa}$. Operating the selection criteria to this high tolerance dramatically reduced the number of false positives encountered.

\section{Results}

\section{Precursor Ion Discovery by Detection of Exact Neutral Loss}

The results of an exact neutral loss experiment acquired from $100 \mathrm{fm}$ of a beta casein digest loaded on column are shown in Figure 2. The first and second traces show the base peak intensity (BPI) chromatograms of the lower and higher collision energy MS acquisitions, respectively. The third trace represents the MS/MS BPI chromatogram, and it can be seen that the instrument has switched only once to this mode of operation during the experiment at 18.8 minutes on the ion at $\mathrm{m} / \mathrm{z}$ 1031.4, suggesting that the doubly charged ion exhibited the specified neutral loss.

Figure 3 shows the lower and higher collision energy spectra at the time of elution for the doubly charged ion at $\mathrm{m} / \mathrm{z}$ 1031.4. The lower collision energy spectrum, acquired with 10 volts applied to the collision cell, contains at least 15 significant, different ions, of which 4 are singly charged, 5 are doubly charged and 6 are triply charged. The corresponding higher collision en- ergy spectrum, acquired with 32 volts applied to the collision cell, shows the complicated mixture of fragment ions derived from all the precursor ions. An expanded view of this spectrum over the range $\mathrm{m} / \mathrm{z}$ 960-1060, shown in Figure 4, reveals that the doubly charged ion at $\mathrm{m} / \mathrm{z} 1031.407$ has potentially produced a doubly charged fragment ion at $\mathrm{m} / \mathrm{z} 982.420$, a difference in $\mathrm{m} / \mathrm{z}$ value of 48.987 . Consequently the instrument switched into the MS/MS product ion mode since this neutral loss was within the specified mass window of $48.989 \pm 0.020$. None of the other product ions in the spectrum have met the criteria to be assigned as a neutral loss.

Having registered the doubly charged ion at $\mathrm{m} / \mathrm{z}$ 1031.4022 as having fragmented with the specified neutral loss the software switched the instrument into the MS/MS mode to record its product ion spectrum, Figure 5. This step is necessary to confirm that the ion assigned as the neutral loss has arisen from the fragmentation of the doubly charged ion at $\mathrm{m} / \mathrm{z} 1031.4022$ and is not a coincidental product ion produced from one of the other peptides present in the source. The product ion spectrum confirmed that the peptide was phosphorylated and also provided sequence information that allowed the phosphorylated peptide to be identified. There are two possible sites for phosphorylation within the peptide sequence and the MS/MS sequence data indicates the position 9 threonine is not modified but the position 3 serine is modified.

Sensitivity of detection by this method of exact neutral loss was determined by analysing a tryptic digest of alpha casein. A series of samples containing 2, 


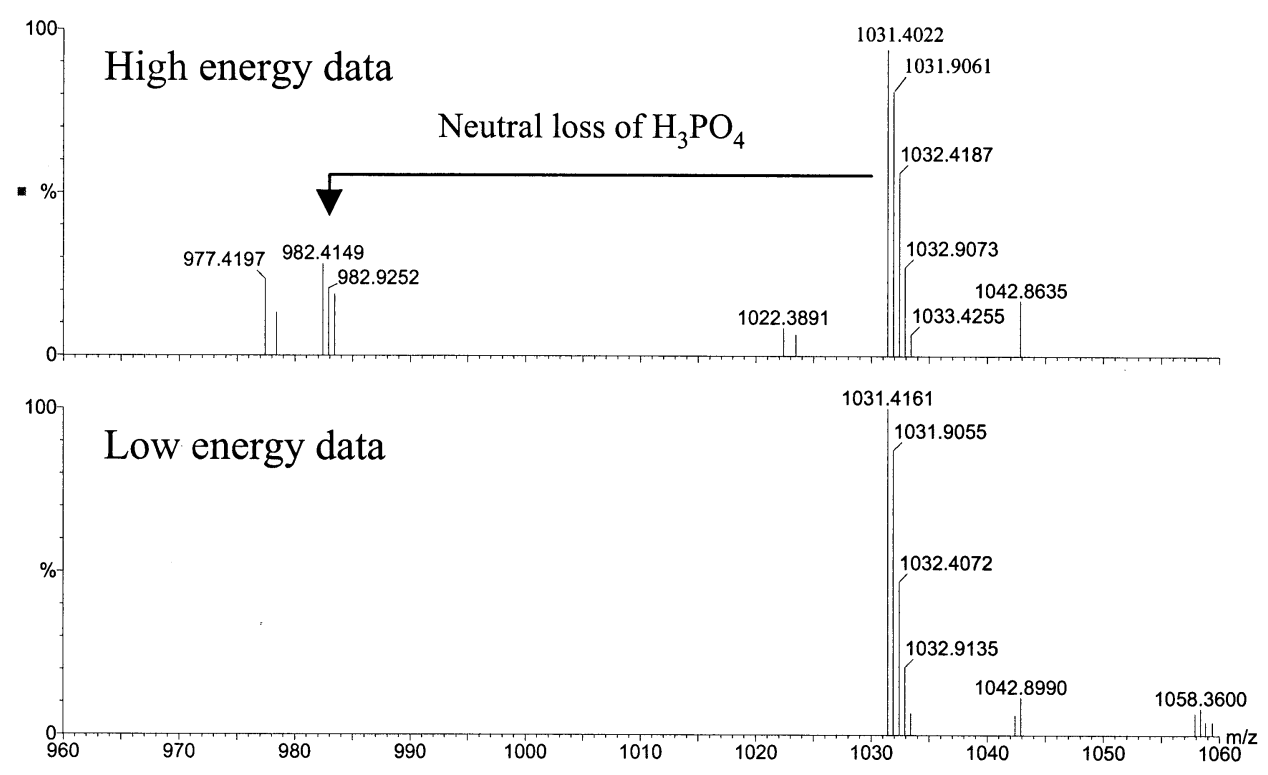

Figure 4. An expanded view of the higher collision energy mass spectrum, shown in Figure 3, over the range $m / z 960-1060$, illustrating the neutral loss of $\mathrm{H}_{3} \mathrm{PO}_{4}(48.989 \mathrm{Da})$ from the doubly charged ion at $m / z 1031.402$.

$5,8,10$ and 20 femtomole respectively were injected on column. Figure 6 shows the product ion spectra obtained from the precursor ion at $m / z 1031.4$ throughout the dilution series. Even for the sample containing only 2 femtomole the exact neutral loss was detected and the mass spectrometer automatically switched to acquire the product ion spectrum.

\section{Precursor Ion Discovery by Detection of Exact Product Ion}

In the case of phosphotyrosine the neutral loss of the $\mathrm{H}_{3} \mathrm{PO}_{4}$ moiety is not commonly observed. However the phosphotyrosine immonium ion at $m / z 216.043$ is observed, and previous studies have shown that precursor ion scanning of this immonium ion can be used to discover peptides containing phosphotyrosine $[19,20]$. In the case of the precursor ion discovery method on the Q-TOF mass spectrometer this characteristic immonium ion is monitored in the higher collision energy data. When detected the mass spectrometer automatically records the product ion spectra of the doubly charged precursor ions observed in the preceding lower collision energy spectrum. It may be necessary to record the product ion spectra of several precursor ions, depending upon the complexity of the peptide mixture eluting.

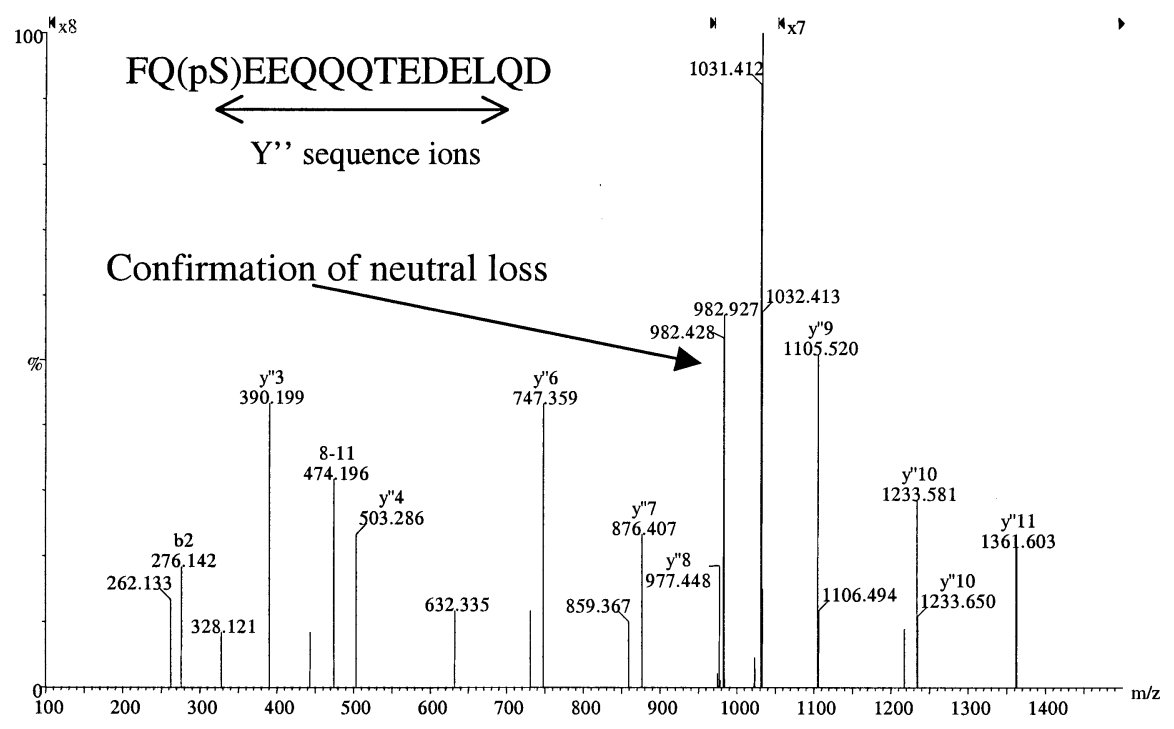

Figure 5. The MS/MS product ion spectrum obtained from the fragmentation of the doubly charged precursor ion at $\mathrm{m} / \mathrm{z}$ 1031.4. The collision cell voltage used in this example was $32 \mathrm{~V}$. 


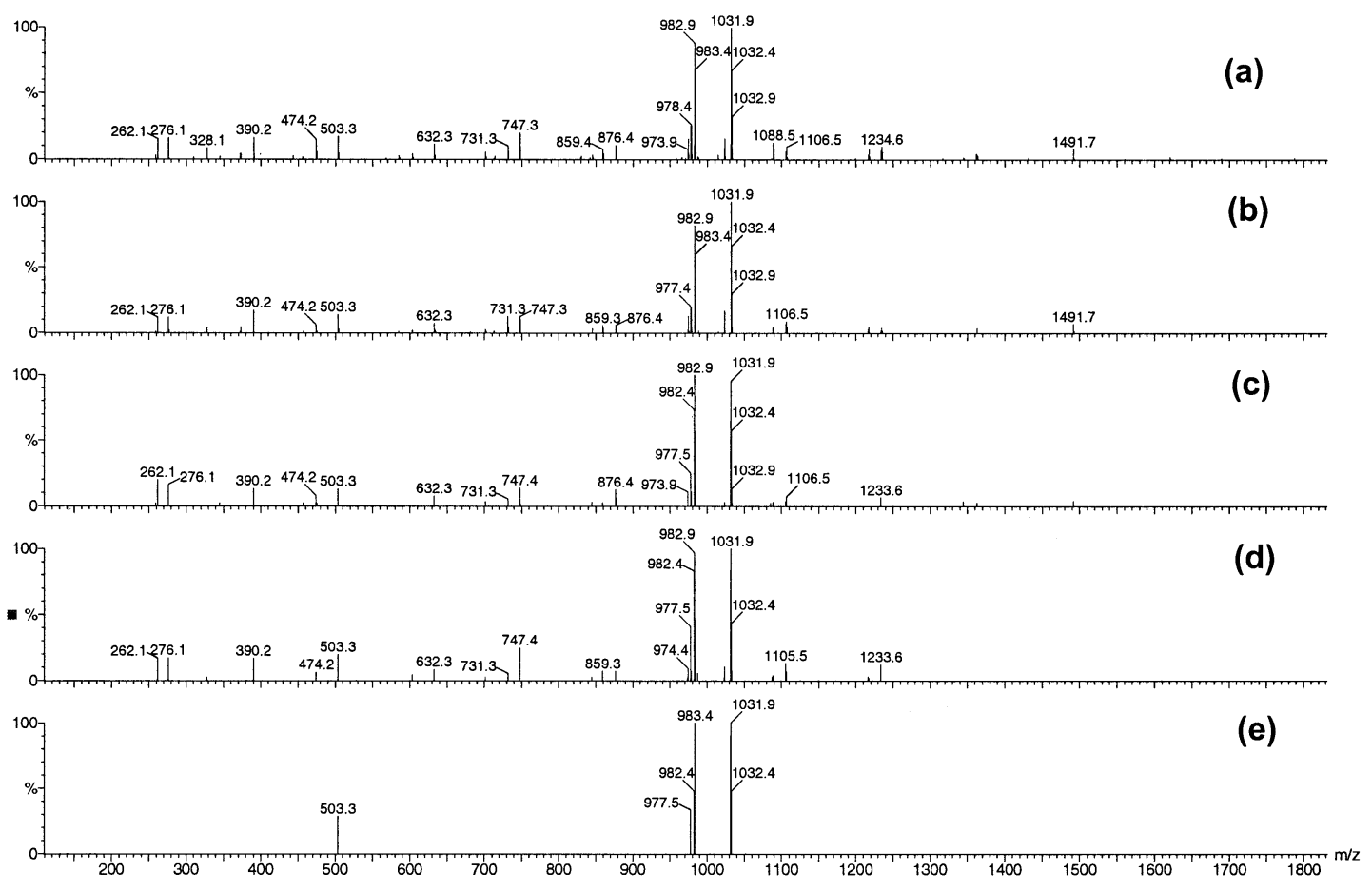

Figure 6. The MS/MS product ion spectra obtained from the doubly charged precursor ion at $\mathrm{m} / \mathrm{z}$ 1031.4 throughout a series of injections of an alpha casein tryptic digest. The series of sample injections contained (a) $20 \mathrm{fmol}$, (b) $10 \mathrm{fmol}$, (c) $8 \mathrm{fmol}$, (d) $5 \mathrm{fmol}$ and (e) 2 fmol respectively.

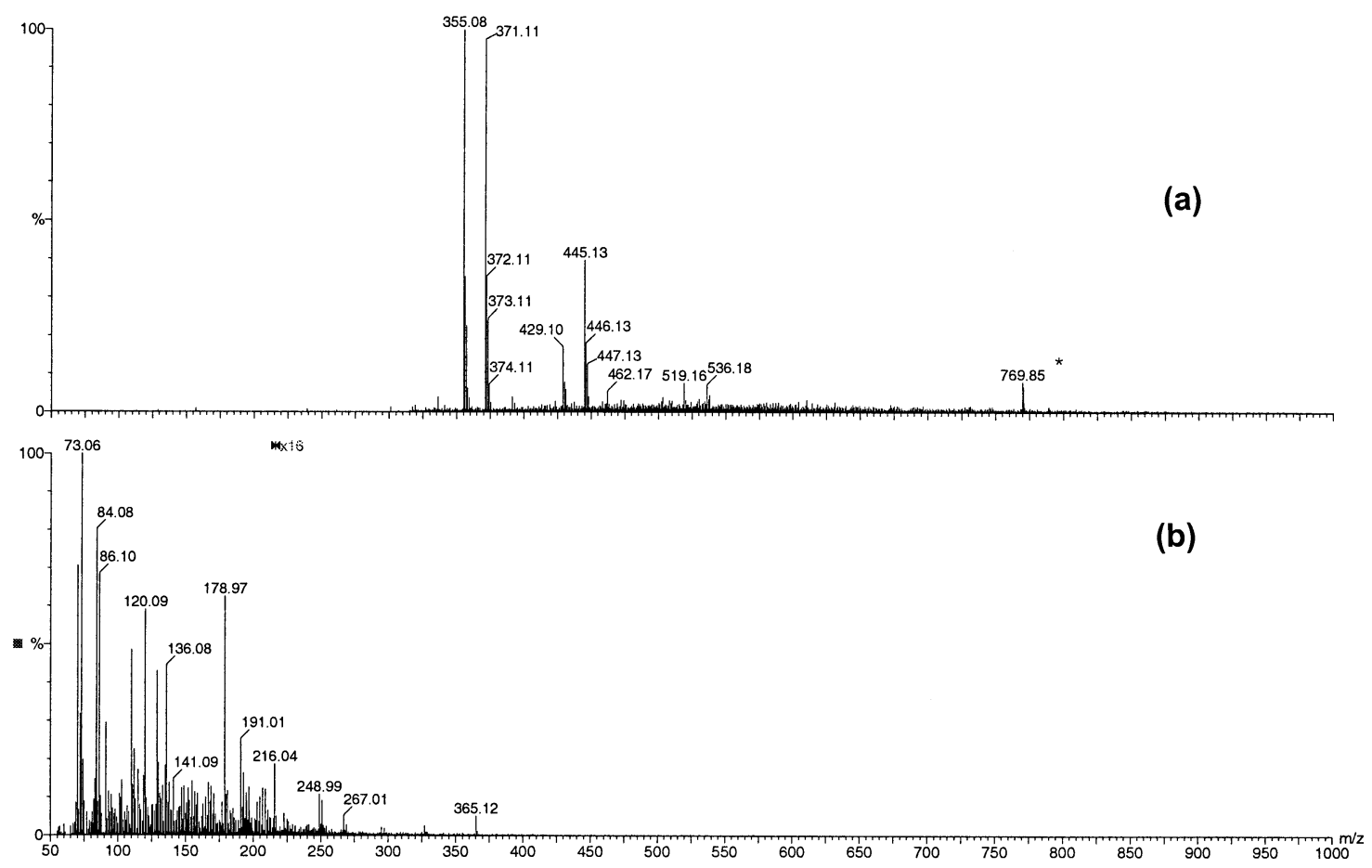

Figure 7. The mass spectra recorded during the LC-MS analysis, using the precursor ion discovery method, from a loading of $10 \mathrm{fmol}$ of the synthetic phosphopeptide TNLSEQ(pY)ADVYR. The doubly charged molecular ion for this peptide can be observed at $\mathrm{m} / z 769.8\left(^{*}\right)$. The upper spectrum was acquired with 7 volts applied to the collision cell (a), and the lower spectrum was acquired with 70 volts applied to the collision cell (b). 


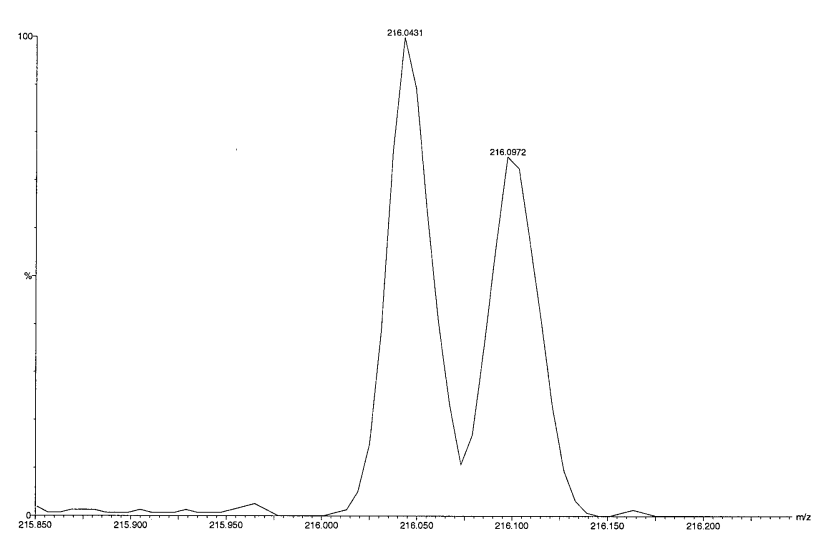

Figure 8. Part of the mass spectrum showing the b2 ion has a mass of 216.098 resolved from the phosphotyrosine immonium ion at $\mathrm{m} / \mathrm{z} 216.043$.

The synthetic phosphopeptide TNLSEQpYADVYR was analyzed by this LC-MS/MS precursor ion discovery method to illustrate the feasibility of this approach for the analysis of tyrosine phosphorylation. In this case $10 \mathrm{fmol}$ of the phosphorylated peptide was injected and analyzed. The spectra shown in Figure 7 were acquired with 7 volts applied to the collision cell in the lower collision energy mode, and 70 volts (which was found to be optimum for generation of the phosphotyrosine immonium ion) in the higher collision energy mode. This particular peptide when fragmented gives rise to a b2 ion that has the same nominal mass as the phosphotyrosine immonium ion, as has been previously reported [21]. This b2 ion has a mass of 216.098, a difference of 1 part in 3900 from that of the phosphotyrosine immonium ion, and Figure 8 shows that these two ions were automatically resolved at the operating resolution (8650 FWHM) of the Q-TOF mass analyzer in these experiments. Both the non-phosphorylated and phosphorylated peptides give rise to the b2 ion, however only the phosphorylated species produces the immonium ion at $\mathrm{m} / \mathrm{z} 216.043$. A mass tolerance of \pm 10 $\mathrm{mDa}$ was set for the precursor ion discovery analysis procedure to ensure only the phosphorylated peptide was detected and not the non-phosphorylated peptide.

A further example of the specificity provided by this approach is shown by the analysis of a mixture containing $25 \mathrm{fmol}$ of the phosphopeptide standard with 250 fmol of a BSA tryptic digest. Figure 9 shows the base peak intensity chromatograms for the lower collision energy and higher collision energy data, the mass chromatogram for $\mathrm{m} / \mathrm{z} 216.043 \pm 0.01$. Figure 10 shows the product ion spectrum of the doubly charged phosphopeptide precursor ion at $m / z$ 769.83. Despite the presence of many high intensity species the mass spectrometer is still able to specifically switch on the phosphorylated peptide and produce an MS/MS spectrum that allows the full sequence of the peptide to be determined.
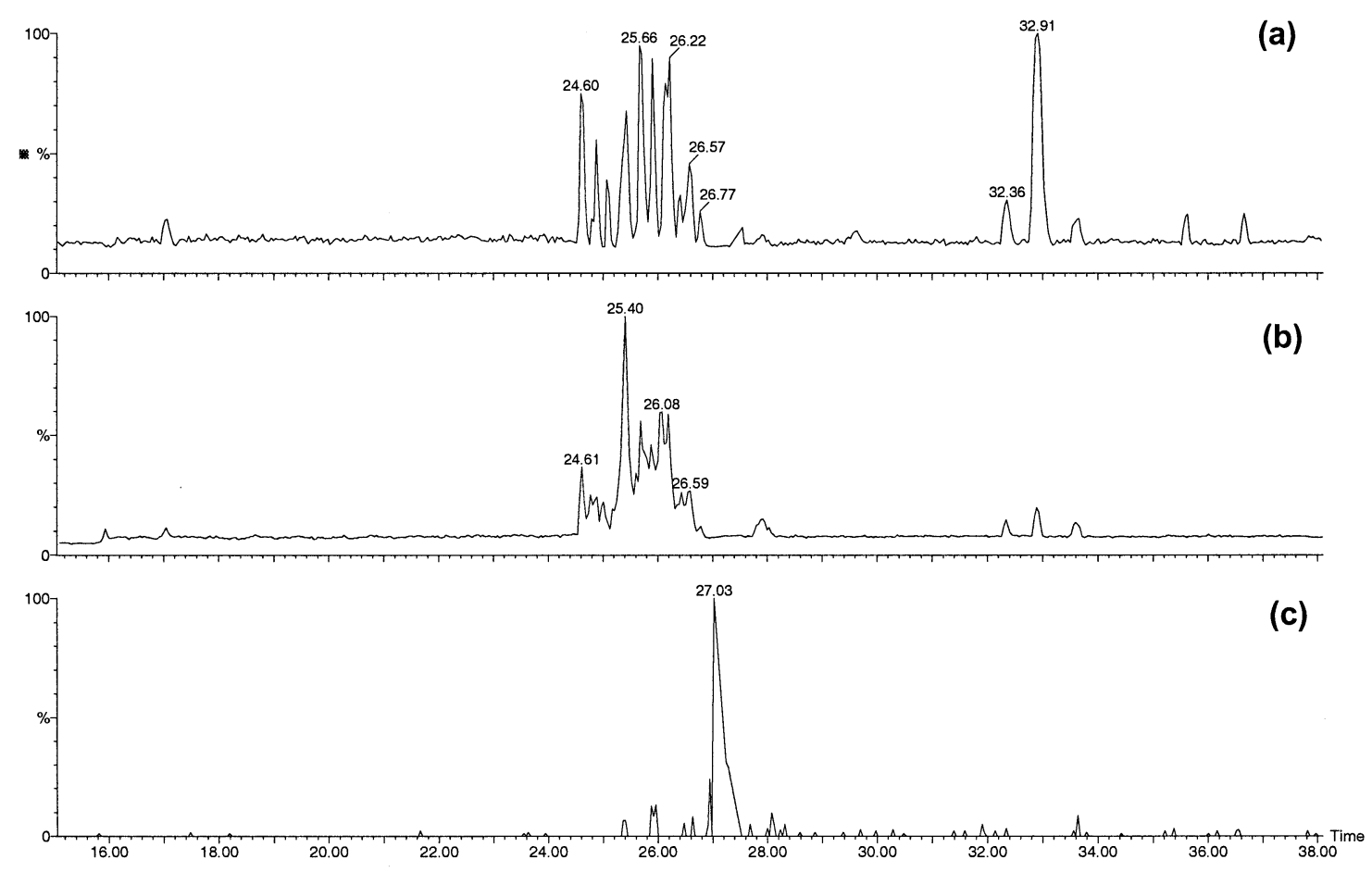

Figure 9. The base peak intensity (BPI) chromatograms for the lower (a) and higher (b) collision energy data, and the selected mass chromatogram for $m / z 216.043 \pm 0.01$ (c). Chromatograms were obtained from LC-MS analysis, using the precursor ion discovery by detection of the low-mass product ion, $m / z 216.043$, to trigger the switch to the product ion MS/MS mode. The sample consisted of a mixture containing $25 \mathrm{fmol}$ of the synthetic phosphopeptide TNLSEQ(pY)ADVYR and $250 \mathrm{fmol}$ of a BSA tryptic digest. 


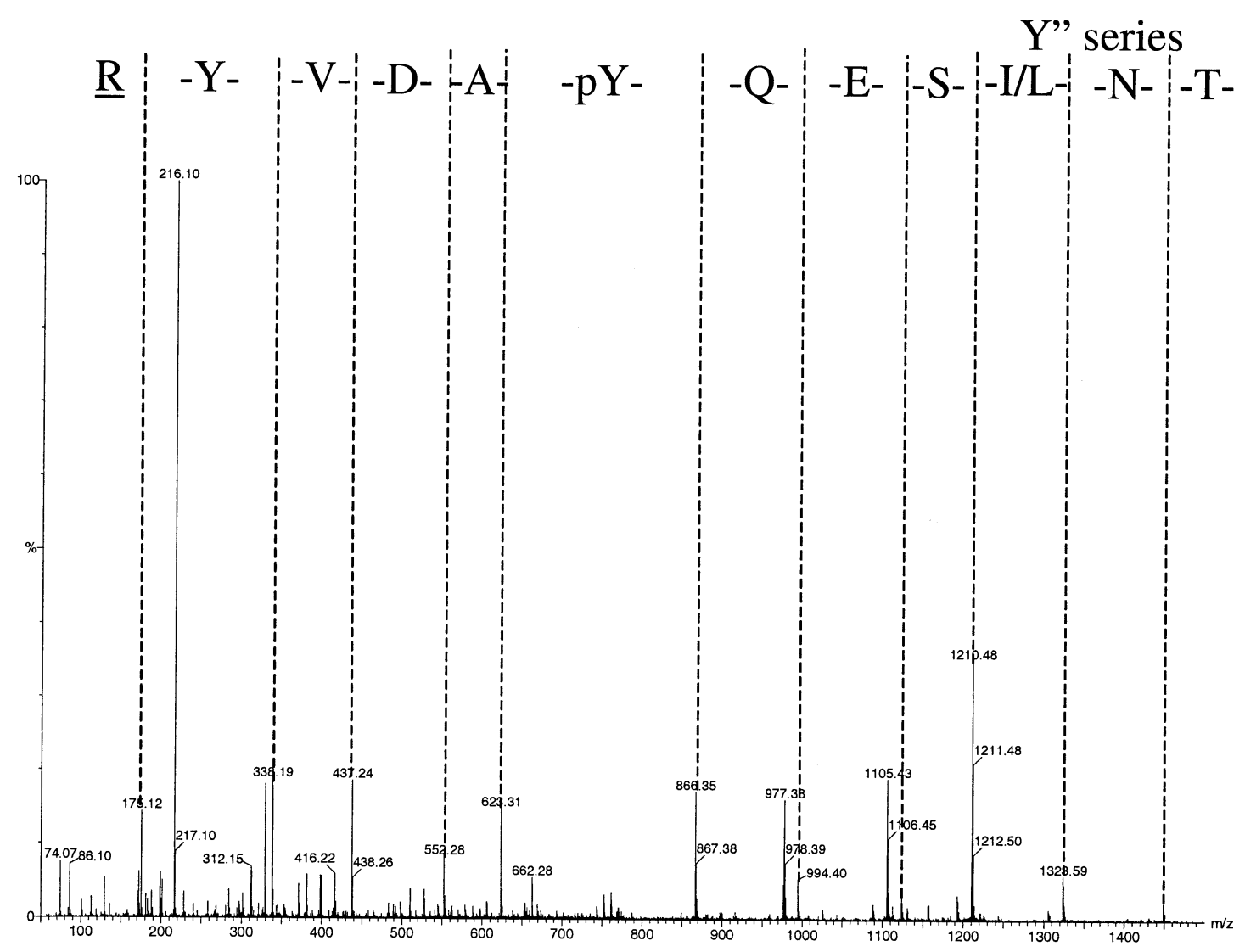

Figure 10. The product ion spectrum produced from fragmentation of the doubly charged phosphopeptide precursor ion at $m / z$ 769.83. The MS/MS spectrum is annotated with the $y^{\prime \prime}$ ion series from the sequence TNLSEQ(pY)ADVYR.

\section{Discussion}

As discussed, a specified neutral loss exposes a precursor and product ion combination and acquisition of the product ion spectrum of that precursor will immediately reveal a successful hit or a false positive. On the other hand a specified product ion is not linked to any particular precursor mass and so discovery takes place by a process of elimination as the instruments switches sequentially through the precursor ion shortlist. It may be necessary to record the product ion spectra of several precursor ions, depending upon the complexity of the peptide mixture eluting. Further to confirmation that the peptide is phosphorylated a product ion spectrum may be needed to provide sequence information of adequate quality to allow the peptide to be identified. The elution profile of the peptide from the HPLC column is typically 9-12 seconds at half height, giving limited time for acquisition of data in the MS/MS mode. This could result in not all precursor ion present being interogated by MS/MS. One method to increase this time is to use a chromatographic technique known as variable flow chromatography or "peak parking." This allows the product ion spectrum from a particular precursor ion to be acquired over a longer period of time. This is achieved by reducing the flow rate from the usual $200 \mathrm{~nL} / \mathrm{min}$ to approximately $20-30 \mathrm{~nL} / \mathrm{min}$, once a particular mass spectral peak of interest is detected by the Q-TOF. The flow from the pumps is diverted to waste, and residual pressure within the system is dissipated through both the analytical column and through a restrictor line. This reduces the flow, leaving a residual flow due to the residual pressure and the electric potential at the spray tip.

Figure 11 shows the mass chromatogram (a) for the doubly charged ion at $\mathrm{m} / \mathrm{z} 598.3$ and the BPI chromatogram (b) obtained from the analysis of an alpha casein tryptic digest by variable flow chromatography. The flow rate was reduced from $200 \mathrm{~nL} / \mathrm{min}$ to approximately 20-30 nL/min during the time that the $m / z 598.3$ ion was detected. This lengthens the elution from approximately 25 seconds at base to approximately 300 seconds, thus increasing the available acquisition time by over an order of magnitude. This would allow an increase in the number of product ion spectra that may be recorded and in the quality of sequence information obtained. This extension to the chromatography run time would only occur if and when the specified product ion is detected. Despite the reduction in the chromatographic flow rate, to allow increased acquisition time on the $\mathrm{m} / \mathrm{z} 598.3$ ion, when the chromato- 

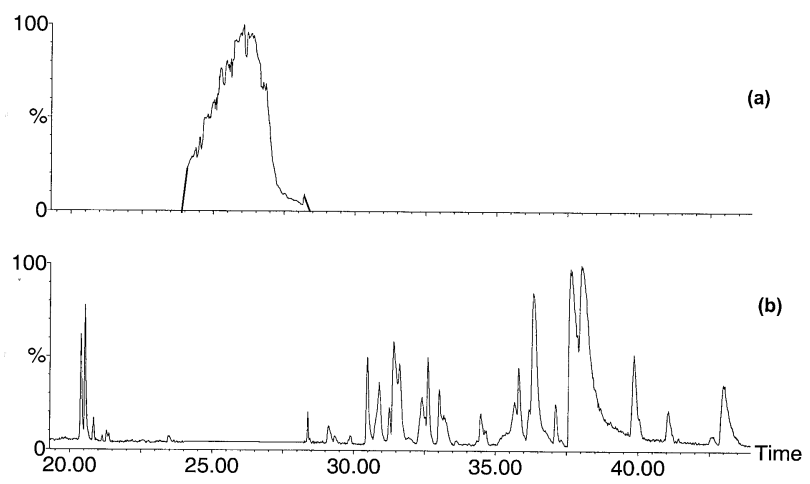

Figure 11. Selected mass chromatogram (a) for the doubly charged ion at $\mathrm{m} / \mathrm{z} 598.3$ and the BPI chromatogram (b) obtained from the analysis of an alpha casein tryptic digest by variable flow chromatography. The flow rate was reduced from $200 \mathrm{~nL} / \mathrm{min}$ to approximately $20-30 \mathrm{~nL} / \mathrm{min}$ during the time that the $\mathrm{m} / \mathrm{z} 598.3$ ion was detected. This results in elution of the ion over a 300 second time window.

graphic elution is resumed at the higher flow rate, there is no degradation of the chromatographic resolution obtained.

\section{Conclusion}

A Q-TOF type instrument has been programmed in such a way that phosphorylated peptides can be discovered and identified within the elution time of liquid chromatography peaks. The method achieves a similar result to that from precursor ion or neutral loss scanning, but without the need to scan the quadrupole mass filter. Instead, the method capitalizes on the ability of the Q-TOF to record mass spectra and product ion spectra quickly, with good sensitivity and with good mass accuracy. The method of analysis is both compatible with and dependent on liquid chromatography for separation of complex mixtures. The method has been demonstrated by searching for the neutral loss of $98 \mathrm{Da}$ $\left(\mathrm{H}_{3} \mathrm{PO}_{4}\right)$ from phosphoserine and phosphothreonine, or for the phosphorylated immonium ion from phosphotyrosine at $\mathrm{m} / \mathrm{z} 216.043$. The sensitivity is such that the neutral loss of $\mathrm{H}_{3} \mathrm{PO}_{4}$ from a beta-casein digest peptide can be detected at levels down to $2 \mathrm{fmol}$ injected on column. The sensitivity of detection of phosphotyrosine is dependent on the complexity of the eluting mixture, and can be as low as $10 \mathrm{fmol}$ in favorable cases. It has also been shown that when the search window for the neutral loss of $\mathrm{H}_{3} \mathrm{PO}_{4}$ from the doubly charged precursor ion is narrowed to $\pm 20 \mathrm{mDa}$ the method can be so specific as to eliminate any false candidate precursor ions. Finally, the method inherently incorporates acquisition of the product ion spectrum of any candidate precursor ions, thereby allowing confirmation of the neutral loss or product ion and providing additional sequence information to assist identification of the protein and assign the site of phosphorylation.

\section{Acknowledgments}

The authors would like to acknowledge the contributions of Bob Bordoli in discussions leading to the development of the methods described in this paper. His presence is missed by all at Micromass.

\section{References}

1. Pappin, D. J. C.; Hojrup, P.; Bleasby, A.J. Curr. Biol. 1993, 3(6), 327-332.

2. Smith, R. D.; Pasa-Tolic, L.; Lipton, M.S.; Jensen, P.K.; Anderson, G.A.; Shen, Y.; Conrads, T.P.; Udseth, H.R.; Harkewicz, R.; Belov, M.E.; Masselon, C.; Veenstra, T.D. Electrophoresis 2001, 22(9), 1652-1668.

3. Hubbard, M. J.; Cohen, P. Trends Biochem. Sci. 1993, 18, 172-177.

4. Hunter, T. Cell 2000, 100, 113-127.

5. Eck, M. J. Current Biol. 1995, 3, 421-424.

6. Schnoelzer, M.; Lehmann, W. D. Int J. Mass Spectrom. Ion Proc. 1997, 169-170, 263-271.

7. Liao, P.; Leykam, J.; Andrews, P. C.; Gage, D. A.; Allison, J. Anal. Biochem. 1994, 219(1), 9-20.

8. Carr, S. A.; Huddleston, M. J.; Annan, R. S. Anal. Biochem. 1996, 239, 180-192.

9. Wilm, M.; Neubauer, G.; Mann, M. Anal. Chem. 1996, 68, 1-8.

10. Covey, T.; Shushan, B.; Bonner, R. In Methods in Protein Sequence Analysis. eds. Jornvall, H.; Hoog, J-O.; Gustavson, A.-M. Bikhauser Verlag: Basel, 1991; pp 249,256.

11. Schlosser, A.; Pipkorn, R.; Bossemeyer, D.; Lehmann, W. D. Anal. Chem. 2001, 73, 170-176.

12. Dawson, J. H.; Guilhaus, M. J. Rapid Commun. Mass Spectrom. 1996, 10, 889-896.

13. Morris, H. R.; Paxton, T.; Dell, A.; Langhorne, J.; Berg, M.; Bordoli, R. S.; Hoyes, J.; Bateman, R. H. Rapid Commun. Mass Spectrom. 1989, 3, 155-159.

14. Bateman, R.; Carruthers, R.; Hoyes, J.; Gilbert, A.; Langridge, J.; Malone, K.; Bordoli, R. Proceedings of 46th ASMS Conference, June 1998, p 42.

15. Whitehouse, C. M.; Gulcicek, E.; Andrien, B.; Banks, F.; Mancini, R. Proceedings of 46th ASMS Conference, June 1998, p 39.

16. Thomson, B. A.; Lock, C. M.; Chernushevich, I. V. Proceedings of 49th ASMS Conference, May 2001.

17. Ekroos, K.; Chernushevich, I.; Simons, K.; Shevchenko, A. Anal. Chem. 2002, 74, 941-949.

18. Hoyes, J. B.; Bateman, R. H.; Wildgoose, J. L. Proceedings of 48 th ASMS Conference, June 2000.

19. Hoffmann, R.; Wachs, W. O.; Berger, R. G.; Kalbitzer, H-R.; Waidelich, D.; Bayer, E.; Wagner-Redeker, W.; Zeppezauer, M. Int. J. Pept. Protein Res. 1995, 45, 26.

20. Lehmann, W. D. Proceedings of 32nd Annual Meeting of the German Mass Spectrometry Society, 1999, p 112.

21. Steen, H; Kuster, B; Mann, M. J Mass Spectrom. 2001, 36, 782-790. 